\title{
The Economic Burden of
} Clostridioides difficile in Denmark: A
Retrospective Cohort Study

\author{
Uffe Christian Braae ${ }^{1 *}$, Frederik Trier Møller ${ }^{1}$, Rikke Ibsen ${ }^{2}$, Steen Ethelberg ${ }^{1,3}$, \\ Jakob Kjellberg ${ }^{4}$ and Kåre Mølbak ${ }^{1,5}$
}

${ }^{1}$ Department of Infectious Disease Epidemiology and Prevention, Statens Serum Institut, Copenhagen, Denmark, ${ }^{2}$ i2minds, Aarhus, Denmark, ${ }^{3}$ Department of Public Health, Faculty of Health and Medical Sciences, University of Copenhagen, Copenhagen, Denmark, ${ }^{4}$ Danish National Institute for Local and Regional Government Research, Copenhagen, Denmark,

${ }^{5}$ Department of Veterinary and Animal Science, University of Copenhagen, Copenhagen, Denmark

\section{OPEN ACCESS}

Edited by:

Mirjana Ratko Jovanovic,

University of Kragujevac, Serbia

Reviewed by:

Julie Abimanyi-Ochom,

Deakin University, Australia

Ana Sabo,

University of Novi Sad, Serbia

*Correspondence: Uffe Christian Braae ucbraae@gmail.com

Specialty section:

This article was submitted to Health Economics,

a section of the journal

Frontiers in Public Health

Received: 17 May 2020 Accepted: 02 November 2020 Published: 26 November 2020

Citation:

Braae UC, Møller FT, Ibsen R, Ethelberg S, Kjellberg J and Mølbak K (2020) The Economic Burden of Clostridioides difficile in Denmark: A

Retrospective Cohort Study. Front. Public Health 8:562957. doi: 10.3389/fpubh.2020.562957
Objectives: The aim of this study was to make a comprehensive economic assessment of the costs of hospital-acquired $C$. difficile infections (CDI).

Methods: We carried out a retrospective matched cohort study utilizing Danish registry data with national coverage to identify CDI cases and matched reference patients without CDI (controls) for economic burden assessment in Denmark covering 2011-2014. Health care costs and public transfer costs were obtained from national registries, and calculated for 1 year prior to, and 2 years after index admission using descriptive statistics and regression analysis.

Results: The study included 12,768 CDI patients and 23,272 matched controls. The total health care cost was significantly larger for CDI cases than controls throughout all periods. During the index admission period, cost was $€ 12,867$ per CDI case compared to $€ 4,522$ ( $p<0.001$ ) for controls, which increased to an average of $€ 31,388$ and $€ 19,512$ $(p<0.001)$ in Year 1 for the two groups, respectively. Excess costs were found both among infections with onset in hospitals and in the community. Diagnosis compatible with complications increased costs to on average $>€ 91,000$ per case. The regression analysis showed that CDI adds a substantial economic burden, but only explains about $1 / 3$ of the crude difference observed in the matched analysis.

Discussion: The major economic impact of hospital-acquired CDI with complications underlines the importance of preventing complications in these patients. Our study provides an informed estimate of the potential economic gain per patient by successful intervention, which is likely to be relatively comparable across countries.

Keywords: economic burden, hospital acquired infections, Clostridium difficile, cohort study, registries, CDI

\section{INTRODUCTION}

Clostridioides difficile is the leading cause of infectious diarrhea in hospitalized patients (1), and occurs regardless of economic development $(2,3)$. C. difficile causes toxin mediated colitis and often associated with a history of antibiotic treatment, increasing age and underlying illness, and exposure to health care systems $(4,5)$. C. difficile infection (CDI) has over the past decades been recognized as an increasing problem with rising mortality rates $(6,7)$. Due to the increase in 
frequency and severity of CDI, assessing the economic burden whereby policy and decision makers can make informed decisions on health care policy, is essential. CDI acquired at hospital or other health care facilities can be sub-classified as either healthcare-onset hospital-acquired (HOHA) or community-onset hospital-acquired (COHA). With the contemporary change in treatment paradigm toward shorter periods of hospitalization and more patients treated in ambulatory care, a change in the epidemiology of CDI with a shift from HOHA to COHA CDI would be expected (8). This hypothesis is corroborated by data from the national surveillance in Denmark (HAIBA annual report 2018, https://www.ssi.dk/-/ media/arkiv/subsites/miba-og-haiba/dokument/haiba_2018-

rsrapport.pdf?la=da), and this possible trend highlights the need for differentiation of the cost estimations for HOHA and COHA CDI. With the implementation of surveillance and more insight into the health burden of CDI, attention is drawn to improved prevention and control of CDI. To determine the cost-effectiveness of potential preventive measures and stimulate research, an economic assessment of the cost associated with CDI is needed. The aim of the present study was to assess the economic burden attributable to CDI during and after hospitalization for both HOHA and COHA CDI, using a population based matched cohort design.

\section{METHODS}

\section{Ethics Statement}

The study was approved under the general agreement for non-interventional database studies between the Danish Data Protection Agency and Statens Serum Institut, reference number 2008-54-0474 and reported according to the STROBE statement (9).

\section{Study Design, Data Sources, and Participants}

We carried out a retrospective matched cohort study utilizing Danish registry data with national coverage to acquire CDI cases and matched reference patients without CDI for economic burden assessment. The sampling population was the entire Danish population from 2011-2014, provided through the Civil Registry System (CRS) containing unique Personal Identification Numbers (PIN), which is linkable to various service records.

We identified cases as patients with a confirmed first episode CDI, through the Hospital-Acquired Infections database (HAIBA). As part of the Danish surveillance system, CDI cases are registered in HAIBA and classified according to the ECDC case definition (10) with a few modifications (11). We distinguished cases as either healthcare-onset (HOHA) if a positive CDI-test was obtained $\geq 48 \mathrm{~h}$ after admission to a hospital and $<48 \mathrm{~h}$ after discharge, or community-onset (COHA) if patients had a positive CDI-test between $48 \mathrm{~h}$ and 4 weeks after health care contact. Inclusion criteria to the case group were all CDI (HOHA and COHA) patients in HAIBA during 2011-2014 with a valid PIN (Figure 1). Patients with community-acquired CDI were excluded, as were patients where no matching control could be identified. All cases were linked with the Danish National Patient Registry (DNPR) (12) to obtain the action diagnosis along with a number of other variables (Supplementary Table 1).

The reference population (hereafter termed controls) were sampled from all admissions recorded during the study period in the DNPR and linked with the CRS to obtain sex and age, but excluding patients from HAIBA with CDI and those without a PIN. We matched each CDI case to two non-CDI controls, but cases with only one match were also retained. The controls were matched according to hospital, region, age, sex, action diagnosis, index year, and index month (that is the month of admission where the first CDI was registered). The action diagnosis was matched on the first three digits and age was matched in groups of intervals of 10 years until $\geq 81$. Furthermore, an outpatient match was additionally performed for COHA patients with a pool of controls with no hospital admission 6 months prior to the end date of their ambulatory treatment. They were matched by sex, age, month, and year of their treatment end date. See the Supplementary Material for the full exclusion criteria and matching description.

\section{Periods for Cost Estimation}

To determine the periods for cost estimations we used the index date in HAIBA as index, but for Year-1, Year 1, and Year 2 we used the dates from DNPR to match with the diagnosis-related groups in the Danish National Cost Database. This database provided the total cost for every in- and outpatient discharged from a public hospital in Denmark based on the patient's actual utilization of hospital services, with Diagnose Related Groups (DRG) for inpatients and Danish Ambulant Grouping System (DAGS) for outpatients. DRG-costs are linked to the discharge date and DAGS-costs are on the date of visit. Due to high mortality in the study population, the number of cases and controls declined over time. We therefore constructed the cost data over time as a gross dataset including all periods for all patients whether they were alive or not, but calculated the average cost of the patients by period, whilst taking the declining population into account. Apart from direct costs, we included derived costs to public transfer from the DREAM database containing all social transfer payments for all citizens in Denmark (13). Cost and employment data from after 2016 was unavailable at the time, so the analysis period, was restricted to 2010-2016, and for the study inclusion period to 2011-2014, to allow for 24 months follow up (Year 1, and Year 2) and 1 year and the pre-index period (Year -1).

\section{Cost Analysis}

Cost data from DRG and DAGS were prices including rehabilitation. Index admission costs for HOHA were from the cost database. For transfer payment (any type of social welfare pay), we calculated the number of months, since we did not have prices for these transfers. Only transfers for people in the workforce were in the data, thus age and pension were unavailable. Complications were identified as the action diagnosis for outpatients and as the main action or secondary diagnosis during admission for inpatients. Patients only contributed cost until the date of death, as cost only occur 


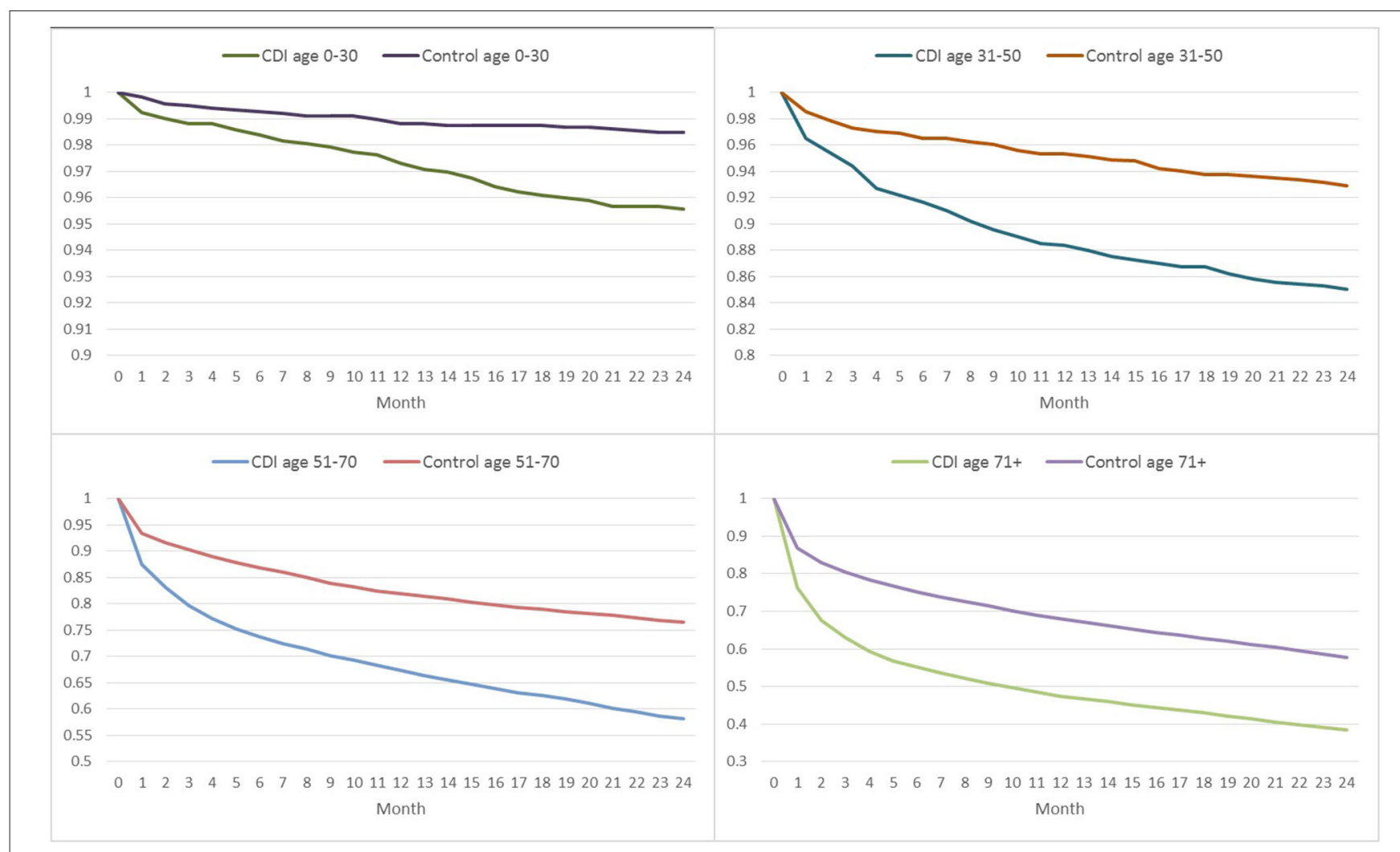

FIGURE 1 | Flowchart illustrating case recruitment from the Hospital-Acquired Infections database (HAIBA) and the number of matched controls identified. CRS, Civil Registry System; PIN, Personal Identification Number; DNPR, Danish National Patient Registry; HOHA, healthcare-onset hospital-acquired; COHA, community-onset hospital-acquired; COCA, community-onset community-acquired.

while patients are alive. The cost were deflated to 2016 prices using the Danish Consumer Price index.

The first analysis included average cost and a test for significant difference between cases and controls. Since cases and controls were matched, we used a bootstrapped $t$-test with no additional explanatory variables in the model. As cost may be affected before onset of CDI, and CDI may result in long term complications, the costs where compared across different cost periods, to be able to compare cost over time. Cost were calculated for pre-index period (Year-1), index, and post-index period (Year 1 and Year 2). The pre-period was divided into two sub-periods, month -12 to -7 and month -6 to -1 . The 24 months post-index period was split into the sub-periods month $1-3$, month 4-6, month 7-9, month 10-12, and month 13-24. Patients were included if they were alive at least part of the subperiod, but excluded in future sub-periods after death occurred. Means of cost data for cases and controls was calculated with $95 \%$ confidence intervals (CI), with all costs adjusted to 2016 prices.

\section{Cost Regression}

To control for residual confounding, we used a regression analysis for specific patients groups in order to determine the added cost of CDI. We used a 2 -step gamma distributed analysis for all health cost (the sum of all types, inpatient somatic, outpatient somatic, inpatient psychiatric, outpatient psychiatric, primary sector, and prescription medication). A gamma distribution link rather than a logarithmic transformation was motivated by the occurrence of 0 's in the cost variables, which did not follow a normal distribution (14). As independent variables we included the Charlson Co-morbidity Index in Year-1, i.e., the period prior to the diagnosis for cases and the corresponding index date for controls. In addition, we adjusted for sex, age, presence of inflammatory bowel disease (IBD), history of drug prescription (received more than four prescription drugs 12 months prior), and whether patients had been in hospital more than 7 days prior to index. This multivariate regression model estimated the total health costs attributed to CDI during the entire period and was run for CDI vs. controls, HOHA CDI vs. controls, and COHA CDI vs. controls. As a sensitivity analysis the model was re-run using data where cases corresponding to $10 \%$ of those with the highest and lowest cost before index and their controls were removed.

\section{Cost Analysis Stratified on Complications}

Cost analysis stratified on complications were run to determine to what extent excess costs in patients could be related to the range of defined clinical complications. Complications were defined as having at least one of the following diagnoses in Year 1 or Year 2 (that is within 24 months after the index date, excluding the index admission): 
TABLE 1 | Descriptive statistics for Clostridioides difficile infection (CDI), healthcare-onset hospital-acquired (HOHA), and community-onset hospital-acquired (COHA) patients and matched controls at index time and prior to inclusion ${ }^{\mathrm{a}}$.

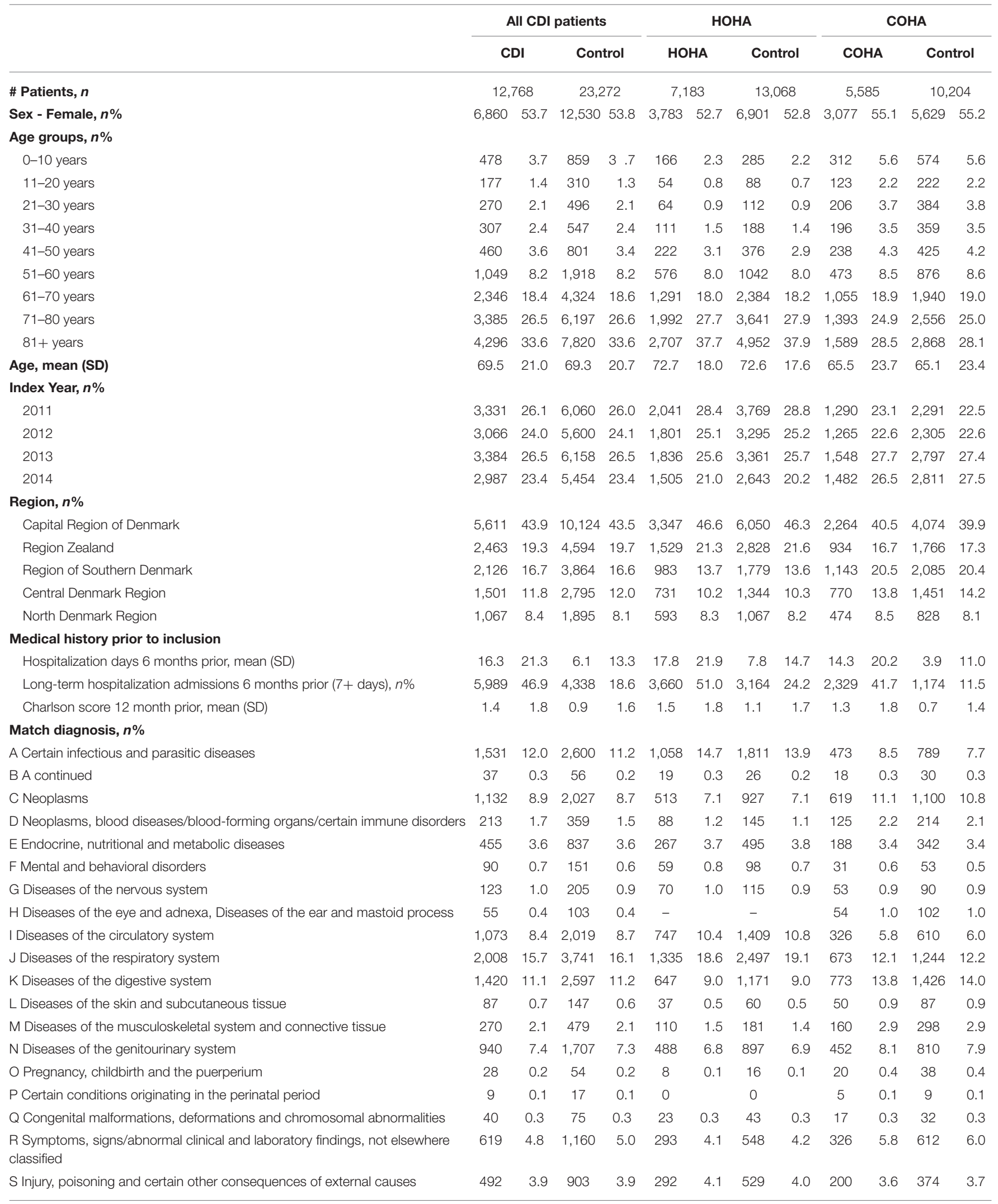




\begin{tabular}{|c|c|c|c|c|c|c|c|c|c|c|c|c|}
\hline TS continued & \multicolumn{4}{|c|}{ All CDI patients } & \multicolumn{4}{|c|}{ HОНА } & \multicolumn{4}{|c|}{ COHA } \\
\hline Z Factors influencing health status and contact with health services & 1,787 & 14.0 & 3,381 & 14.5 & 896 & 12.5 & 1,675 & 12.8 & 891 & 16.0 & 1,706 & 16.7 \\
\hline \multicolumn{13}{|l|}{ IBD 12 month prior, $n \%$} \\
\hline K50_12M_prior & 160 & 1.3 & 150 & 0.6 & 53 & 0.7 & 65 & 0.5 & 107 & 1.9 & 85 & 0.8 \\
\hline K52_12M_prior & 365 & 2.9 & 240 & 1.0 & 186 & 2.6 & 143 & 1.1 & 179 & 3.2 & 97 & 1.0 \\
\hline A0_12M_prior & 831 & 6.5 & 435 & 1.9 & 417 & 5.8 & 307 & 2.3 & 414 & 7.4 & 128 & 1.3 \\
\hline Share any of the above IBD & 1,468 & 11.5 & 919 & 3.9 & 688 & 9.6 & 555 & 4.2 & 780 & 14.0 & 364 & 3.6 \\
\hline \multicolumn{13}{|l|}{ Number of prescription drug ATC-codes 7 digits 12 month prior, $n \%$} \\
\hline 0 & 313 & 2.5 & 991 & 4.3 & 195 & 2.7 & 442 & 3.4 & 118 & 2.1 & 549 & 5.4 \\
\hline 0 & 313 & 2.5 & 991 & 4.3 & 195 & 2.7 & 442 & 3.4 & 118 & 2.1 & 549 & 5.4 \\
\hline $1-5$ & 2,605 & 20.4 & 6,744 & 29.0 & 1405 & 19.6 & 3240 & 24.8 & 1200 & 21.5 & 3,504 & 34.3 \\
\hline $6-9$ & 3,190 & 25.0 & 6,325 & 27.2 & 1818 & 25.3 & 3565 & 27.3 & 1372 & 24.6 & 2,760 & 27.0 \\
\hline $10+$ & 6,660 & 52.2 & 9,212 & 39.6 & 3765 & 52.4 & 5821 & 44.5 & 2895 & 51.8 & 3,391 & 33.2 \\
\hline \multicolumn{13}{|l|}{ Number of prescription drug ATC-codes 3 digits 12 month prior, $n \%$} \\
\hline 0 & 313 & 2.5 & 991 & 4.3 & 195 & 2.7 & 442 & 3.4 & 118 & 2.1 & 549 & 5.4 \\
\hline $1-5$ & 3,066 & 24.0 & 7,670 & 33.0 & 1661 & 23.1 & 3737 & 28.6 & 1405 & 25.2 & 3,933 & 38.5 \\
\hline $6-9$ & 3,880 & 30.4 & 7,253 & 31.2 & 2236 & 31.1 & 4229 & 32.4 & 1644 & 29.4 & 3,024 & 29.6 \\
\hline $10+$ & 5,509 & 43.1 & 7,358 & 31.6 & 3091 & 43.0 & 4660 & 35.7 & 2418 & 43.3 & 2,698 & 26.4 \\
\hline
\end{tabular}

a Distributions do not always amount to $100 \%$, as cells with $<5$ observations are not listed due to anonymity requirements.

${ }^{b} / C D-10=K 50, K 51, K 52, A 0$.

septicaemia, dehydration, renal failure, ileus, hypotension, shock, thromboembolic episodes, toxic megacolon, bowel perforation, colectomy, and isolation regimes. Cases with complications were allocated to the complication group for the whole study period and then stratified regardless of when the complication occurred. For a detailed description of the methodology of the matching and the cost analysis, please see the Supplementary Material.

\section{RESULTS}

\section{Matching Results and Population Descriptive}

The study included 12,768 patients with hospital-acquired CDI and 23,272 matched controls (Table 1). Of the CDI cases, 7,183 were identified as HOHA and 5,585 cases as COHA, with 13,068 and 10,204 matched controls for each group, respectively. Most CDI patients were females (53.7\%), and the mean age was 69.5 years with $33.6 \%$ of the cases being $>80$ years of age. The distribution of CDI cases across index years 2011-2014 were approximately equal (range: 3,384-3,036). Common diagnoses were diseases of the respiratory system (15.7\%), factors influencing health status and contact with health services (14.0\%), and certain infectious and parasitic diseases (12.0\%).

Prior to the index date, cases and matched controls were incomparable on a number of parameters. Cases had on average spent 16.3 days in hospital compared with 6.1 days spent among the controls. The share of long-term hospitalization admissions (7+ days) 6 months prior was 46.9 and $18.6 \%$ among the cases and the controls, respectively. The Charlson score 12 months prior to inclusion was on average 1.4 and 0.9 for cases and controls, respectively. Among the cases, $11.5 \%$ had a diagnosis of IBD 12 months prior to inclusion, whereas $3.9 \%$ of the controls had IBD. Taken together, even with a careful matching procedure based on underlying disease codes, cases of CDI had an excess of severe illness and were hospitalized longer, and had higher frequencies of IBD before index date. Thus, matching did not account for the differences in morbidity between the cases and controls.

\section{Complications and Mortality}

Typical complications for COHA and HOHA were septicaemia, renal failure, dehydration, and isolation regimes (Supplementary Table 2). During Year 1, 8.1\% cases had more than one complication, with septicaemia and renal failure causing most days in hospital among both COHA and HOHA cases. The complications among CDI patients accounted for a 


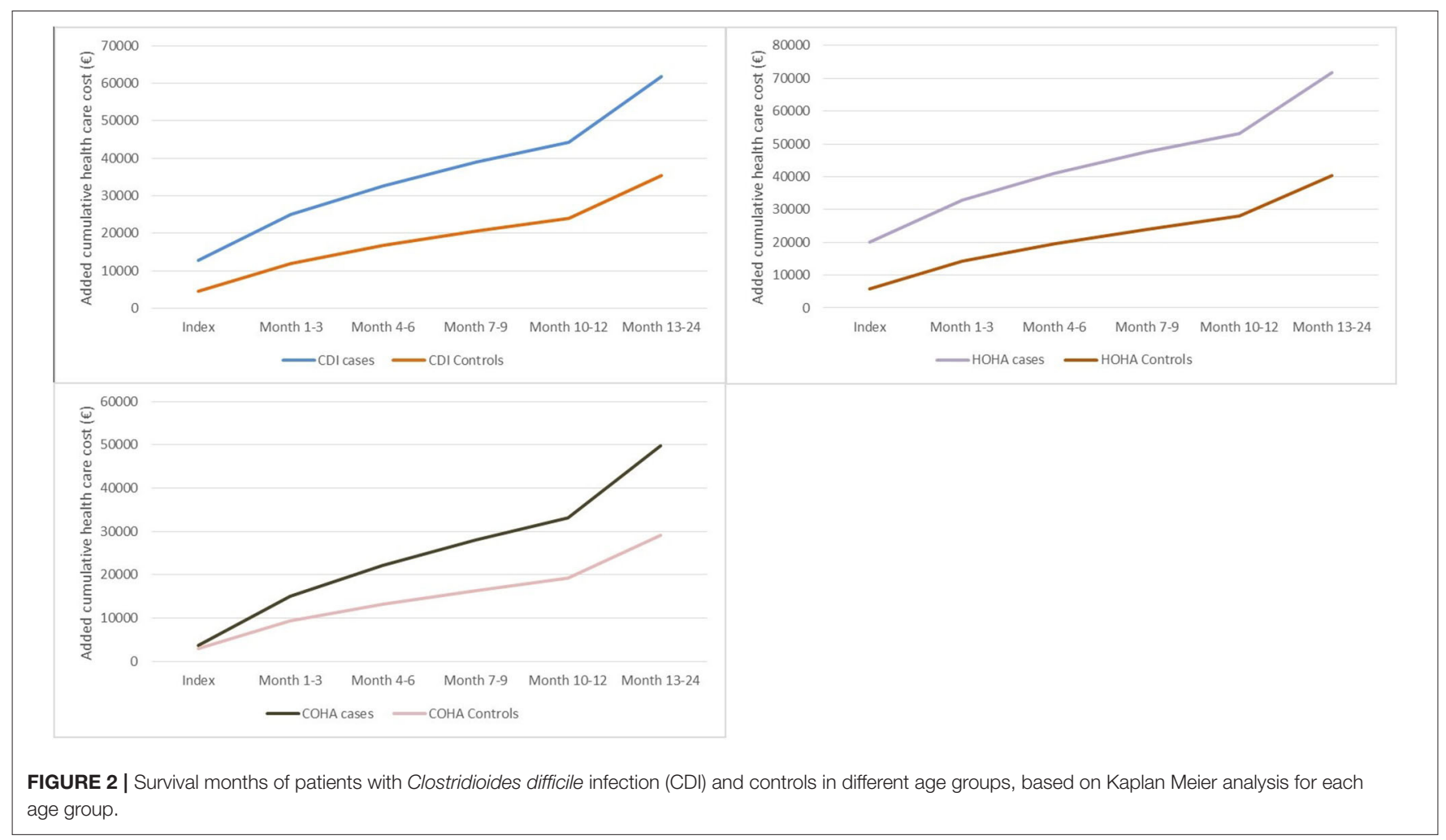

large share of the days spent in hospital. COHA CDI patients were on average hospitalized 17.7 days during Year 1, with 34.6\% attributed to complications, and 8.0 days during Year 2, with $32.2 \%$ attributed to complications. HOHA CDI patients were in comparison hospitalized on average 21.7 days during Year 1, with $32.6 \%$ attributed to complications, and 10.5 days during Year 2, with $32.4 \%$ due to complications (Supplementary Table 3).

The mortality among CDI patients was high, especially among the age group $71+$ years, where $38 \%$ survived until 24 months, compared to $58 \%$ of the matched controls (Figure 2). The difference in survival between CDI patients and the controls became less apparent with declining age. In general, $\mathrm{COHA}$ cases had better survival than HOHA cases, e.g., a $21.7 \%$ survival among $71+$ HOHA compared with $31.0 \%$ among COHA after 72 months (Supplementary Figures 1, 2). A similar magnitude of difference in survival was seen among the matched controls which may indicate that the relative mortality was independent of onset of CDI.

\section{Cost of C. difficile Infections}

The total health care cost was significantly larger for CDI cases compared to the controls throughout all periods studied, including Year-1 before diagnosis (Table 2). A full breakdown of all costs for CDI, COHA, and HOHA, is available in the Supplementary Tables 5.1-5.9. During month -12 to -7 the average cost of CDI cases was $€ 8,700$ compared to $€ 6,073$ ( $p$ $<0.001$ ) for the controls, which increased to $€ 21,795$ compared to $€ 10,188$ ( $p<0.001)$ for the two groups during month -6 to -1 . At index, cost was $€ 12,867$ for cases compared to $€ 4,522$ $(p<0.001)$ for controls. In Year 1 , the cost was $€ 31,388$ for CDI and $€ 19,512$ for the controls $(p<0.001)$, and $€ 17,590$ and $€ 11,260$ ( $p<0.001)$ for the two groups, respectively in Year 2. During the index period and Year 1 and 2, CDI cases spent on average 1.1 month longer on public transfer income compared to the controls $(p<0.001)$. We found increased costs both for COHA and for HOHA CDI cases compared with the matched controls (Table 2). At index the mean cost of a COHA CDI case was $€ 3,704$ compared to $€ 2,996$ ( $p<0.001)$ for a control, and $€ 16,640$ vs. $€ 9,850$ ( $p<0.001)$ during Year 2 for cases and controls, respectively. HOHA CDI cases on average were estimated to cost $€ 19,992$ at index compared to $€ 5,714$ ( $~ p$ $<0.001)$ for controls. During Year 2 the costs were estimated to $€ 18,553$ and $€ 12,523$ ( $p<0.001)$ for cases and controls, respectively. Based on the cumulative health care cost from index to Year 2, HOHA CDI cases were more costly with a mean economic burden of $>€ 70,000$, whereas, a COHA CDI case had a mean economic burden of $\sim € 50,000$ (Figure 3).

\section{Economic Burden of CDI}

The largest share of the economic burden was related to complications in combination with CDI, which after 2 years on average resulted in an economic burden of more than $€ 91,000$ per case compared to $\sim € 40,000$ among the matched controls. In comparison, cases without complications incurred an economic burden after 2 years of $\sim € 41,000$ whereas the matched controls incurred an economic burden of $\sim € 32,000$ (Figure 4).

The regression model (see Tables $\mathbf{3}, \mathbf{4}$ for the parameters) showed that over a 2 -year period, $33 \%$ of the economic burden 
TABLE 2 | CDI health care costs of all CDI patients, COHA, HOHA, and their matched controls.

\begin{tabular}{|c|c|c|c|c|c|c|c|c|c|c|c|c|c|c|c|}
\hline \multirow[b]{4}{*}{ Period } & \multicolumn{5}{|c|}{ Health cost CDI } & \multicolumn{5}{|c|}{ Health cost COHA } & \multicolumn{5}{|c|}{ Health cost HOHA } \\
\hline & \multicolumn{2}{|c|}{ \# Patients } & \multicolumn{3}{|c|}{ Total health cost } & \multicolumn{2}{|c|}{ \# Patients } & \multicolumn{3}{|c|}{ Total health cost } & \multicolumn{2}{|c|}{ \# Patients } & \multicolumn{3}{|c|}{ Total health cost } \\
\hline & CDI & Control & CDI & Control & $P$-value* & COHA & Control & COHA & Control & $P$-value* & HOHA & Control & НОНА & Control & $P$-value* \\
\hline & $n$ & $n$ & $€$ & $€$ & & $n$ & $n$ & $€$ & $€$ & & $n$ & $n$ & $€$ & $€$ & \\
\hline Year-1 (-1 to -12 month) & 12,768 & 23,272 & 30,494 & 16,261 & $<0.001$ & 5,585 & 10,204 & 29,613 & 12,873 & $<0.001$ & 7,183 & 13,068 & 31,180 & 18,906 & $<0.001$ \\
\hline Month-12 to-7) & 12,786 & 23,272 & 8,700 & 6,073 & $<0.001$ & 5,585 & 10,204 & 9,114 & 5,327 & $<0.001$ & 7,183 & 13,068 & 8,377 & 6,655 & $<0.001$ \\
\hline Month -6 to -1$)$ & 12,786 & 23,272 & 21,795 & 10,188 & $<0.001$ & 5,585 & 10,204 & 20,499 & 7,546 & $<0.001$ & 7,183 & 13,068 & 22,802 & 12,251 & $<0.001$ \\
\hline Index cost** & 12,768 & 23,272 & 12,867 & 4,522 & $<0.001$ & 5,585 & 10,204 & 3,704 & 2,996 & $<0.001$ & 7,183 & 13,068 & 19,992 & 5,714 & $<0.001$ \\
\hline Month 1-3 & 11,846 & 22,134 & 12,102 & 7,498 & $<0.001$ & 5,571 & 9,868 & 11,244 & 6,267 & $<0.001$ & 6,275 & 12,266 & 12,864 & 8,487 & $<0.001$ \\
\hline Month 4-6 & 9,196 & 19,873 & 7,742 & 4,716 & $<0.001$ & 4,502 & 9,092 & 7,187 & 3,887 & $<0.001$ & 4,694 & 10,781 & 8,274 & 5,416 & $<0.001$ \\
\hline Month 7-9 & 8,366 & 18,901 & 6,200 & 3,826 & $<0.001$ & 4,140 & 8,768 & 5,861 & 3,203 & $<0.001$ & 4,226 & 10,133 & 6,533 & 4,366 & $<0.001$ \\
\hline Month 10-12 & 7,883 & 18,184 & 5,344 & 3,472 & $<0.001$ & 3,938 & 8,515 & 5,081 & 2,928 & $<0.001$ & 3,945 & 9,669 & 5,607 & 3,951 & $<0.001$ \\
\hline Year 2 (month 13-24) & 7,512 & 17,580 & 17,590 & 11,260 & $<0.001$ & 3,782 & 8,305 & 16,640 & 9,850 & $<0.001$ & 3,730 & 9,275 & 18,553 & 12,523 & $<0.001$ \\
\hline
\end{tabular}

${ }^{\star} P$-value from $t$-test and bootstrapping.

**Index admissions are calculated from the cost database for HOHA. Since COHA is partly outpatient onset, their index admission is from DRG.

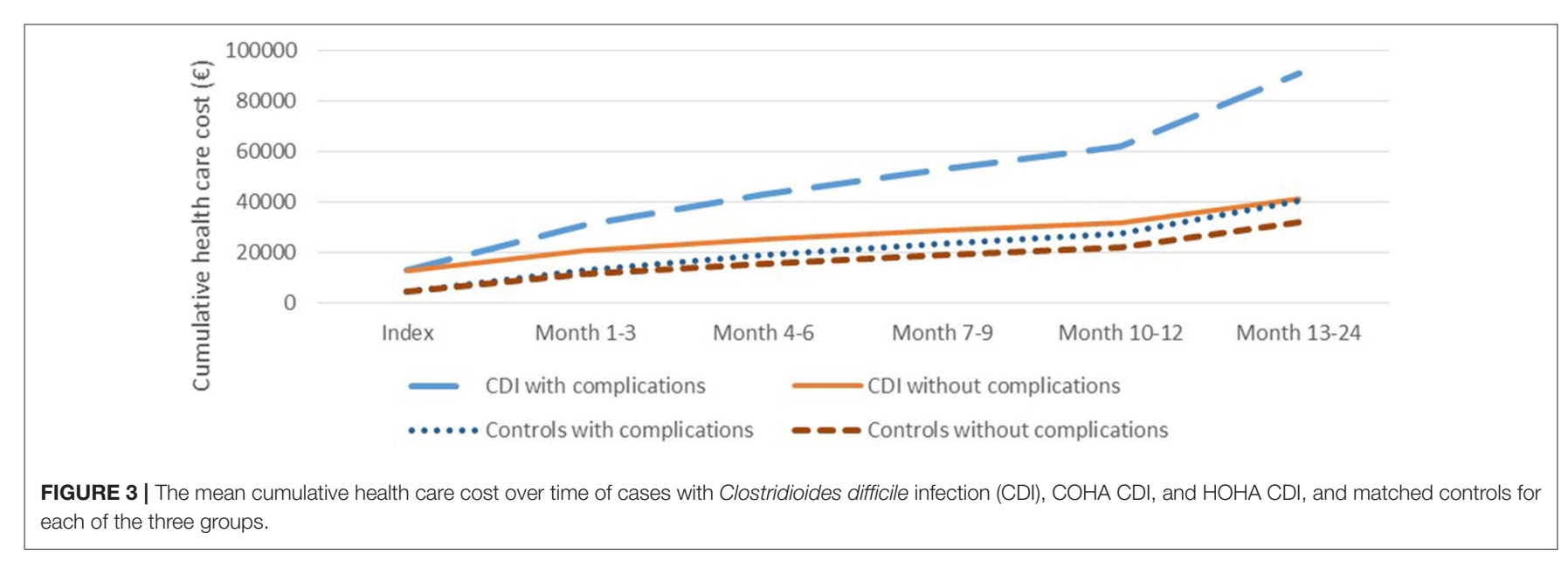

among patients with CDI could be ascribed to CDI. Among patients with HOHA CDI, $37 \%$ of the economic burden could be explained by CDI, whereas $23 \%$ of the economic burden could be attributed to CDI among COHA CDI patients. Table 3 shows the estimated health care costs of typical patients groups during different periods and the estimated potential gained monetary value by preventing CDI. The largest attribution to cost by CDI was observed during index and the 1st year of disease. From the estimated cost in specific patients groups, the model predicted that in patients with severe disease, a smaller proportion of the cost could be attributed to CDI in comparison to "healthier" patients (Table 5). The sensitivity analysis showed that there was no significant impact by removing the $10 \%$ of the data covering outliers in either end (data not shown).

\section{DISCUSSION}

Although cases and controls were carefully matched on several variables including age and diagnosis, we found considerable differences in clinical parameters and hospital costs between cases and controls even before the diagnosis of CDI. This underscores that CDI is a challenge of the frailest patients, and that it is critical to address confounding in epidemiological and economic studies of CDI. It is, however, also possible that some of the difference in Year-1 can be ascribed to a delayed diagnosis of CDI. Interestingly the cost difference was less during the months -12 to -7 than during the months -6 to -1 leading to the index; potentially indicating that CDI had manifestations well before diagnosis in some patients. To address confounding, we conducted a regression analysis, which confirmed that CDI adds a substantial economic burden, but only explains about $1 / 3$ of the crude difference observed in the matched analysis. The conducted sensitivity analysis showed that the model accounted well for the cost differences between cases and controls prior to index. The cost difference between cases and controls were more than four times higher in CDI cases with complications than cases without complications. The association between clinical complications and excess costs adds additional weight to the plausibility of the results. 


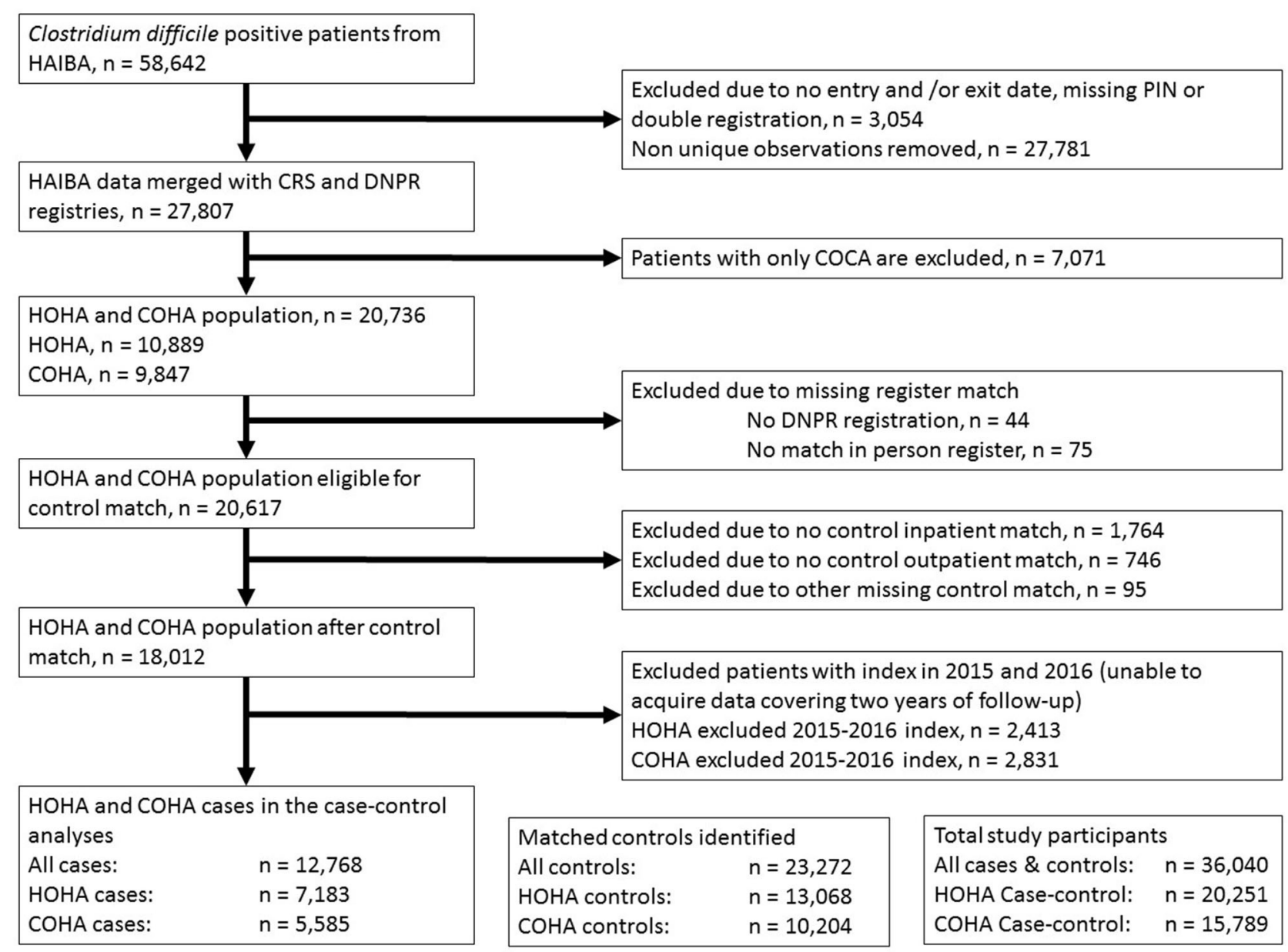

FIGURE 4 | The mean cumulative health care cost over time of cases with Clostridioides difficile infection (CDI) with and without complications and the matched controls.

We found excess costs both in HOHA and COHA CDI patients, but we also found that the costs of COHA was less than among HOHA. While this difference was expected, we believe that our study is the first to address this question. The differences in the economic health care burden of HOHA and COHA underlines the importance of acknowledging the distinction of the two groups in economic health care burden analyses, in particular because COHA will be relatively more important as health care reform progresses toward more ambulatory care.

There is limited consensus about the definition of CDI, populations studied, the designs, and range of cost included. This complicates an overall comparison between our study and previous published literature. A recent review of the economic burden of CDI from different countries found that the attributable mean CDI costs ranged from $\$ 8,911$ to $\$ 30,049$ for hospitalized patients (2014 USD) (15). Although direct comparisons are difficult, our estimates for the health care costs of CDI patients appear well outside this range as the average CDI case in Denmark, with the inclusion of the 2 year postperiod, cost $\sim € 61,800$ in 2016 , equivalent to $\$ 68,000$ USD.
However, higher estimates are often seen in health economics when using high quality registry data with broad coverage due to the potential capability of capturing more of the actual costs (16).

A recent retrospective analysis using individual-level data from from Apr. 1, 2005 to Mar. 31, 2015, in Ontario Canada health databases, found the median costs attributable to C. difficile infection to be $\$ 1051$ for that associated with long-term care facilities, \$13 249 for community-associated infection and $\$ 11917$ for ACH-associated/community-onset infection (17).

A study from Germany estimating both direct and indirect cost of CDI in hospitalized patients found CDI to cost $€ 18,460$ (18), which is comparable to the index cost of $€ 19,992$ for HOHA patients found in this study. A study from Australia that investigated CDI in a population aged 45 years and up (19), found that patients with cardiovascular disease cost on average $\$ 17,947$ $\pm 2,611$ with CDI and $\$ 7,825 \pm 46.7$ without CDI (difference of AUS $\$ 10,122 \sim € 6,230)$. This is comparable to the difference in cost at index $(€ 8,345)$ between a CDI case $(€ 12,867)$ and a control $(€ 4,522)$ in our study. 
TABLE 3 | Regression total health cost including all patients alive at least part of year 1 (including index date).

\begin{tabular}{|c|c|c|c|c|c|c|c|}
\hline Population & $N$ & Year 1 & \multicolumn{5}{|c|}{ Total health cost Year 1} \\
\hline $\operatorname{CDI}(N)$ & 12,768 & CDI vs. control & 0.47 & 0.01 & 0.44 & 0.49 & $<0.001$ \\
\hline & & Charlson Comorbidity Index (CCI) & 0.14 & 0.00 & 0.14 & 0.15 & $<0.001$ \\
\hline & & Gender (male) & 0.18 & 0.01 & 0.16 & 0.20 & $<0.001$ \\
\hline & & Age & -0.07 & 0.00 & -0.08 & -0.07 & $<0.001$ \\
\hline HOHA, all included & & HOHA, all included & & & & & \\
\hline & & Charlson Comorbidity Index (CCl) & 0.09 & 0.00 & 0.08 & 0.10 & $<0.001$ \\
\hline & & Gender (male) & 0.15 & 0.01 & 0.12 & 0.18 & $<0.001$ \\
\hline & & Age & -0.16 & 0.00 & -0.17 & -0.15 & $<0.001$ \\
\hline COHA, all included & & COHA, all included & & & & & \\
\hline $\mathrm{COHA}(N)$ & 5,585 & COHA vs. control & 0.36 & 0.02 & 0.33 & 0.40 & $<0.001$ \\
\hline \multirow[t]{3}{*}{ Control $(N)$} & 10,204 & Controlled for & & & & & \\
\hline & & Charlson Comorbidity Index (CCI) & 0.21 & 0.01 & 0.19 & 0.22 & $<0.001$ \\
\hline & & Gender (male) & 0.17 & 0.02 & 0.14 & 0.20 & $<0.001$ \\
\hline
\end{tabular}

Nanwa et al. (20) conducted an incidence-based propensityscore matched cohort study to evaluate costs attributable to hospital-acquired CDI from the healthcare payer perspective. They found that hospital-acquired CDI was associated with worse clinical outcomes in patients compared to clinical outcomes in matched uninfected patients. Similarly, our study reported higher mortality among cases compared to the matched controls (Figure 2). Nanwa et al. (20) also reported that the attributable costs were greatest during the index hospitalization, but decreased over time albeit higher costs persisted compared to matched non-CDI patients. This is comparable to the results presented here for hospitalized patients, but for COHA CDI patients the largest economic burden was incurred in Month 1-3 after treatment.

McGlone et al. (21) developed an economic computational model to determine the annual cost of healthcare-acquired CDI in the Unites States. The model incorporated hospital-acquired CDI associated costs in regards to hospital, third-party payer, and societal perspectives. Most costs incurred were during a patient's primary CDI episode, with an estimated cost of as much as $\$ 12,607$ (2011 USD). With the regression models developed during the present study, we can estimate the economic burden of various patient groups with hospital-acquired CDI, and the potential to develop the model further to quantify the total economic burden of all hospital-acquired CDI patients in Denmark exist.

Our study is subject to limitations. Although clinical outcomes are representative of the clinical settings in other high-income countries, data on direct costs and public transfer are to a large degree specific to the Danish society. Nonetheless, the results do have general international applicability due to the uniqueness of the data quality, the large range of outcomes, the large sample size, and the potential to be modified to other settings and used in modeling burden of CDI in other countries. We had no detailed clinical data on comorbidity, which represents another limitation. However, the use of a comorbidity score and adjustment for history of medications is thought to have adjusted for confounding. This is supported by the observation that IBD (which results in increased sampling of fecal specimens and therefore an increased chance of diagnosis of CDI) were not associated with increased costs in the regression analysis. Finally, complications were defined based on data from administrative registries and not a detailed clinical assessment, which was beyond the scope of this study. 
TABLE 4 | Regression total health cost including all patients alive all of Year 1 and at least part of Year 2.

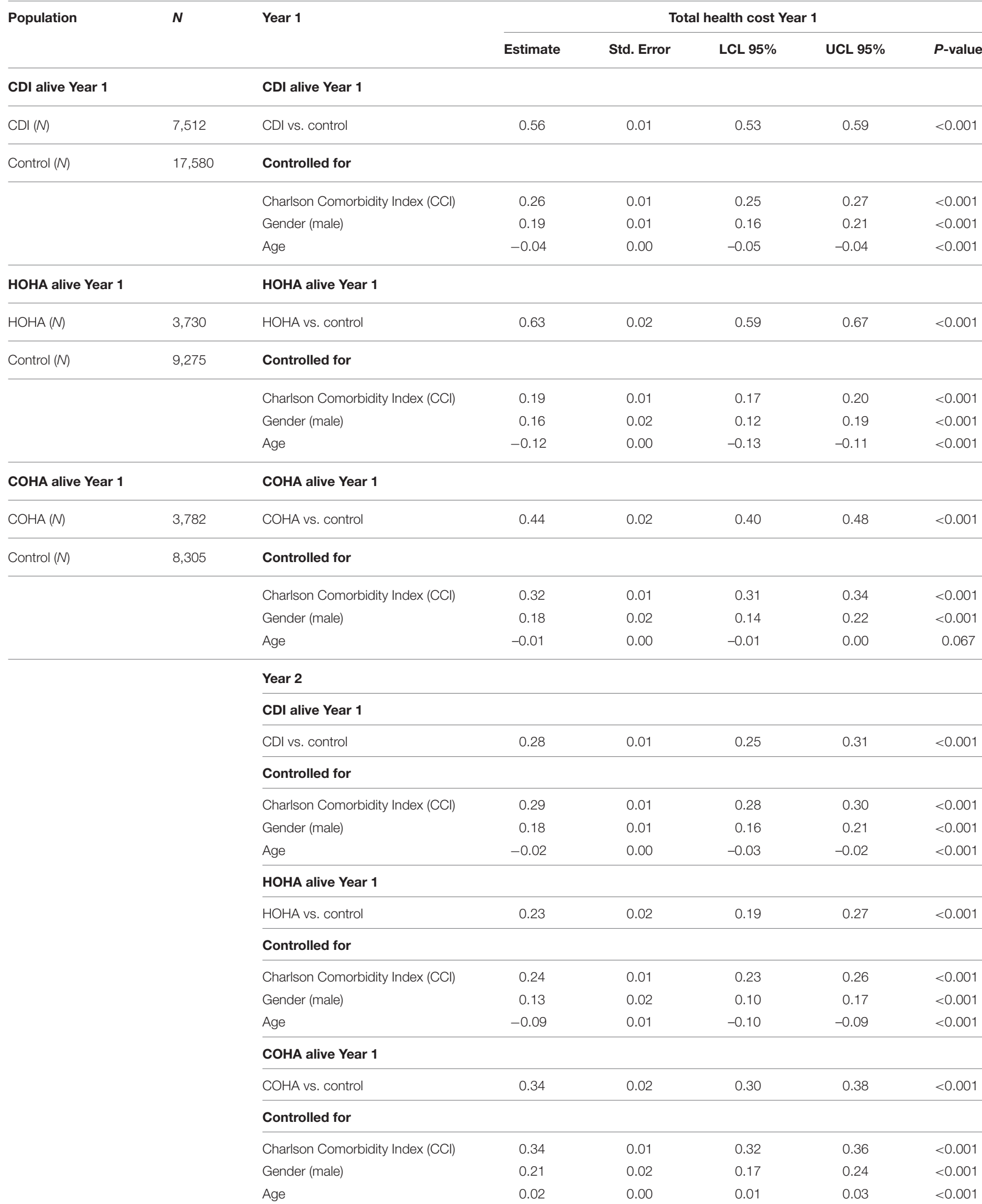


TABLE 5 | Total cost and the added cost of CDI among selected patient groups from CDI, CDI HOHA, and CDI COHA.

\begin{tabular}{|c|c|c|c|c|c|c|}
\hline & \multicolumn{3}{|c|}{ Per case economic burden, $€$} & \multicolumn{3}{|c|}{ Monetary gain per case of CDI prevention, $€(\%)$} \\
\hline & $\begin{array}{c}\text { Index and } \\
\text { part of Year } \\
1\end{array}$ & Year 1 & Year 2 & $\begin{array}{l}\text { Index and part } \\
\text { of Year } 1\end{array}$ & Year 1 & Year 2 \\
\hline \multicolumn{7}{|l|}{ CDI patients } \\
\hline Women aged $51-60$ & 24,948 & 22,343 & 6,983 & $\begin{array}{c}8,214 \\
(33)\end{array}$ & $\begin{array}{c}8,362 \\
(37)\end{array}$ & $1,105(16)$ \\
\hline Women aged 51-60 with a co-morbidity score 2 & 31,373 & 33,773 & 11,406 & $\begin{array}{l}10,329 \\
(33)\end{array}$ & $12,640(37)$ & $1,805(16)$ \\
\hline $\begin{array}{l}\text { Woman aged } 51-60 \text { with a co-morbidity score } 2 \text { and } 7+\text { days in } \\
\text { hospital prior }\end{array}$ & 48,810 & 58,422 & 17,018 & $\begin{array}{l}20,055 \\
(41)\end{array}$ & $24,329(42)$ & $4,654(27)$ \\
\hline $\begin{array}{l}\text { Woman aged } 51-60 \text { with a co-morbidity score } 2 \text {, ATC5, and } 7 \text { +days in } \\
\text { hospital prior }\end{array}$ & 42,291 & 51,886 & 23,477 & $\begin{array}{c}6,681 \\
(16)\end{array}$ & $\begin{array}{c}8,384 \\
(16)\end{array}$ & $2,258(10)$ \\
\hline \multicolumn{7}{|l|}{ HОНА CDI patients } \\
\hline Women aged $51-60$ & 38,892 & 35,419 & 9,113 & $\begin{array}{c}14,372 \\
(37)\end{array}$ & $15,373(43)$ & $1,474(16)$ \\
\hline Women aged 51-60 with a co-morbidity score 2 & 45,384 & 48,556 & 13,879 & $\begin{array}{l}16,772 \\
(37)\end{array}$ & $21,075(43)$ & $2,245(16)$ \\
\hline Women aged 51-60 with 7+days in hospital prior and ATC5 & 44,274 & 44,378 & 15,604 & $\begin{array}{c}10,261 \\
(23)\end{array}$ & $11,281(25)$ & $\begin{array}{l}1,294 \\
(8)\end{array}$ \\
\hline Women aged 51-60 with 7+days in hospital prior, ATC5, and IBD & 36,419 & 35,405 & 16,882 & $\begin{array}{c}3,509 \\
(10)\end{array}$ & $\begin{array}{c}2,650 \\
(7)\end{array}$ & $\begin{array}{c}-2,216 \\
(-13)\end{array}$ \\
\hline \multicolumn{7}{|l|}{ COHA CDI patients } \\
\hline Women aged $41-50$ & 15,827 & 14,265 & 5,798 & $\begin{array}{c}3,576 \\
(23)\end{array}$ & $\begin{array}{c}3,732 \\
(26)\end{array}$ & $\begin{array}{l}845 \\
(15)\end{array}$ \\
\hline Women aged 41-50 with a co-morbidity score 1.5 & 20,174 & 25,293 & 8,804 & $\begin{array}{c}4,558 \\
(23)\end{array}$ & $\begin{array}{c}6,617 \\
(26)\end{array}$ & $1,283(15)$ \\
\hline Women aged $41-50$ with a co-morbidity score 1.5 and ATC5 & 25,358 & 27,201 & 15,145 & $\begin{array}{c}5,825 \\
(23)\end{array}$ & $\begin{array}{c}7,213 \\
(27)\end{array}$ & $2,035(13)$ \\
\hline Women aged $41-50$ with a co-morbidity score 1.5 and IBD & 23,157 & 24,373 & 11,197 & $\begin{array}{c}7,858 \\
(34)\end{array}$ & $\begin{array}{c}8,162 \\
(33)\end{array}$ & $2,151(19)$ \\
\hline
\end{tabular}

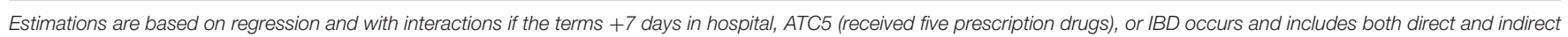
costs. The period "part of Year 1" includes patients that died during this period. A proportionately identical difference was observed for men (data not shown).

In conclusion, our study estimates the attributable economic burden of hospital-acquired CDI in Danish patients and provides an informed estimate of the potential economic gain per patient by successful intervention. We emphasize the need to include COHA CDI in order to make comprehensive estimates of the overall economic burden of CDI on health care systems. At present, only few national surveillance systems have the capacity to disentangle HOHA from COHA, and often COHA cases may be ignored because the onset is in the community where they may not be categorized as hospital-acquired cases. Furthermore, the results highlight the importance of identifying and preventing complications in patients with hospital-acquired CDI, and the need to investigate strategies to prevent CDI in susceptible patients.

\section{DATA AVAILABILITY STATEMENT}

The datasets presented in this article are not readily available because the authors confirm that, for approved reasons, some access restrictions apply to the data underlying the findings. We used population-register based data including personal identifiers. To access this data it is necessary to apply to the Danish National Board of Health by completing an extensive data approval application.

\section{AUTHOR CONTRIBUTIONS}

RI and JK generated and analyzed the data. UB, FM, SE, and KM interpreted the data. UB wrote the first manuscript draft. All authors critically reviewed the manuscript.

\section{FUNDING}

This study was conducted with financial support from the Merck Sharp \& Dohme Corporation. The funder had no role in study 
design, data collection and analysis, decision to publish, or preparation of the manuscript.

\section{ACKNOWLEDGMENTS}

We acknowledged the collaboration with the network of Danish clinical microbiology laboratories provided data for the national surveillance and the HAIBA group at Statens

\section{REFERENCES}

1. Leffler DA, Lamont JT. Clostridium difficile infection. N Engl J Med. (2015) 372:1539-48. doi: 10.1056/NEJMra1403772

2. He M, Miyajima F, Roberts P, Ellison L, Pickard DJ, Martin MJ, et al. Emergence and global spread of epidemic healthcare-associated Clostridium difficile. Nat Genet. (2013) 45:109-13. doi: 10.1038/ng.2478

3. Roldan GA, Cui AX, Pollock NR. Assessing the burden of Clostridium difficile infection in low- and middle-income countries. J Clin Microbiol. (2018) 56:e01747-17. doi: 10.1128/JCM.01747-17

4. Carroll KC, Bartlett JG. Biology of Clostridium difficile: implications for epidemiology and diagnosis. Annu Rev Microbiol. (2011) 65:501-21. doi: 10.1146/annurev-micro-090110-102824

5. McFarland LV, Mulligan ME, Kwok RY, Stamm WE. Nosocomial acquisition of Clostridium difficile infection. $N$ Engl J Med. (1989) 320:204-10. doi: 10.1056/NEJM198901263200402

6. Ricciardi R, Rothenberger DA, Madoff RD, Baxter NN. Increasing prevalence and severity of Clostridium difficile colitis in hospitalized patients in the United States. Arch Surg. (2007) 142:624-31; discussion 631. doi: 10.1001/archsurg.142.7.624

7. Bauer MP, Notermans DW, van Benthem BH, Brazier JS, Wilcox MH, Rupnik $\mathrm{M}$, et al. Clostridium difficile infection in Europe: a hospital-based survey. Lancet. (2011) 377:63-73. doi: 10.1016/S0140-6736(10)61266-4

8. Gubbels S, Nielsen KS, Sandegaard J, Mølbak K, Nielsen J. The development and use of a new methodology to reconstruct courses of admission and ambulatory care based on the Danish National Patient Registry. Int J Med Inform. (2016) 95:49-59. doi: 10.1016/j.ijmedinf.2016.08.003

9. von Elm E, Altman DG, Egger M, Pocock SJ, Gøtzsche PC, Vandenbroucke JP, et al. The Strengthening the Reporting of Observational Studies in Epidemiology (STROBE) statement: guidelines for reporting observational studies. Lancet. (2007) 370:1453-7. doi: 10.1016/S0140-6736(07)61602-X

10. Kuijper EJ, Coignard B, Tüll P, ESCMID Study Group for Clostridium difficile, EU Member States, European Centre for Disease Prevention and Control. Emergence of Clostridium difficile-associated disease in North America and Europe. Clin Microbiol Infect. (2006) 12:2-18. doi: 10.1111/j.1469-0691.2006.01580.x

11. Chaine M, Gubbels S, Voldstedlund M, Kristensen B, Nielsen J, Andersen LP, et al. Description and validation of a new automated surveillance system for Clostridium difficile in Denmark. Epidemiol Infect. (2017) 145:2594-602. doi: 10.1017/S0950268817001315

12. Schmidt M, Schmidt SA, Sandegaard JL, Ehrenstein V, Pedersen L, Sørensen HT. The Danish National Patient Registry: a review of content, data quality, and research potential. Clin Epidemiol. (2015) 7:449-90. doi: 10.2147/CLEP.S91125

13. Hjollund NH, Larsen FB, Andersen JH. Register-based follow-up of social benefits and other transfer payments: accuracy and degree of completeness
Serum Institut, in particular Sophie Gubbels, Jens Nielsen, and Manon Chaine.

\section{SUPPLEMENTARY MATERIAL}

The Supplementary Material for this article can be found online at: https://www.frontiersin.org/articles/10.3389/fpubh. 2020.562957/full\#supplementary-material

in a Danish interdepartmental administrative database compared with a population-based survey. Scand J Public Health. (2007) 35:497-502. doi: 10.1080/14034940701271882

14. Buntin MB, Zaslavsky AM. Too much ado about two-part models and transformation? Comparing methods of modeling Medicare expenditures. $J$ Health Econ. (2004) 23:525-42. doi: 10.1016/j.jhealeco.2003.10.005

15. Nanwa N, Kendzerska T, Krahn M, Kwong JC, Daneman N, Witteman W, et al. The economic impact of Clostridium difficile infection: a systematic review. Am J Gastroenterol. (2015) 110:511-9. doi: 10.1038/ajg.2015.48

16. Pereira JA, McGeer A, Tomovici A, Selmani A, Chit A. Incidence and economic burden of Clostridioides difficile infection in Ontario: a retrospective population-based study. CMAJ Open. (2020) 8:16-25. doi: 10.9778/cmajo.20190018

17. Busse R, Schreyögg J, Smith PC. Variability in healthcare treatment costs amongst nine EU countries - results from the HealthBASKET project. Health Econ. (2008) 17:1-8. doi: 10.1002/hec.1330

18. Heimann SM, Vehreschild JJ, Cornely OA, Wisplinghoff H, Hallek M, Goldbrunner R, et al. Economic burden of Clostridium difficile associated diarrhoea: a cost-of-illness study from a German tertiary care hospital. Infection. (2015) 43:707-14. doi: 10.1007/s15010-015-0810-x

19. Chen Y, Glass K, Liu B, Korda RJ, Riley TV, Kirk MD. Burden of Clostridium difficile infection: associated hospitalization in a cohort of middle-aged and older adults. Am J Infect Control. (2017) 45:508-11. doi: 10.1016/j.ajic.2016.12.006

20. Nanwa N, Kwong JC, Krahn M, Daneman N, Lu H, Austin PC, et al. The economic burden of hospital-acquired Clostridium difficile infection: a population-based matched cohort study. Infect Control Hosp Epidemiol. (2016) 37:1068-78. doi: 10.1017/ice.2016.122

21. McGlone SM, Bailey RR, Zimmer SM, Popovich MJ, Tian Y, Ufberg P, et al. The economic burden of Clostridium difficile. Clin Microbiol Infect. (2012) 18:282-9. doi: 10.1111/j.1469-0691.2011.0 3571.x

Conflict of Interest: RI was employed by the company i2minds.

The remaining authors declare that the research was conducted in the absence of any commercial or financial relationships that could be construed as a potential conflict of interest.

Copyright (c) 2020 Braae, Møller, Ibsen, Ethelberg, Kjellberg and Mølbak. This is an open-access article distributed under the terms of the Creative Commons Attribution License (CC BY). The use, distribution or reproduction in other forums is permitted, provided the original author(s) and the copyright owner(s) are credited and that the original publication in this journal is cited, in accordance with accepted academic practice. No use, distribution or reproduction is permitted which does not comply with these terms. 